\title{
SHOT NOISE AND PROXIMITY EFFECT IN SUPERCONDUCTOR-NORMAL-METAL HETEROSTRUCTURES
}

\author{
W. BELZIG, J. BÖRLIN AND C. BRUDER \\ Department of Physics and Astronomy, Klingelbergstr. 82, University of Basel, \\ 4056 Basel, Switzerland \\ YU. V. NAZAROV \\ Department of Applied Physics, Delft University of Technology, The Netherlands
}

\begin{abstract}
Current noise provides useful information on correlations important for transport properties of mesoscopic systems. Of particular interest, both experimentally and theoretically, are heterostructures of normal metals and superconductors. We use an extended Keldysh-Green's function approach to calculate the current correlations fully accounting for the superconducting proximity effect. The shot noise in diffusive wires shows a reentrant behaviour, similar to the reentrance effect of the conductance. At intermediate energies the effective charge (the ratio of the differential noise and differential conductance) is suppressed below the value of the Cooper pair charge 2e due to higher correlations. In superconducting heterostructures with more than two normal leads, we address the question of the sign of correlations between currents in different terminals. We show that positive crosscorrelations are a generic feature in these structures, which should be easily observable experimentally.
\end{abstract}

\section{Introduction}

The current in mesoscopic structures fluctuates in time. Due to the coherent nature of the transport, these fluctuations provide information on the quantum physics of the underlying transport mechanism (see Ref. 1 for a summary). For example, measuring the current noise $S_{I}$ in a tunnel junction allows to extract the effective charge of the carriers involved. The effective charge of a tunnel junction is defined similar to Fano factor by the ratio $q_{\mathrm{eff}}^{\mathrm{tun}}=S_{I} / 2 I$, where $I$ is the average current. This has been used to determine effective charges of $e / 3$ and $e / 5$ in the fractional quantum Hall regime. For non-opaque junctions the definition of an effective charge only makes sense in reference to something known. For example, in superconducting heterostructures one usually takes the normal state as reference point and we may define an effective charge in reference to that. In this way an effective charge $2 e$ in the case of Andreev reflection for diffusive contact has been predicted theoretically and determined experimentally 2 . 4 .

It is also possible to measure nonlocal current-current correlations, the so-called crosscorrelations. A fermionic version of the Hanbury-Brown and Twiss experiment was performed and showed negative crosscorrelation 2 . These originate from the Pauli exclusion principle, which leads to a noiseless stream of incoming particles at zero temperature. The electrons are scattered at a beam splitter one by one. Obviously an electron leaving in one lead can not leave in the other, therefore the time-dependent fluctuations in the two leads are anticorrelated, thus leading to negative crosscorrelations. This argument has been put on solid ground by Büttiker $\mathbf{6}$, who has shown that this holds for transport of fermions in arbitrary 


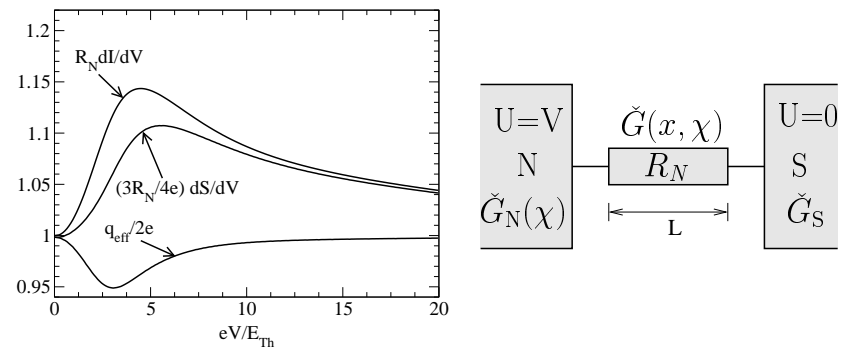

Figure 1. Transport properties of a diffusive proximity wire. The differential conductance $d I / d V$ normalized by the normal state resistance shows the usual reentrance behaviour around voltages of the order of the Thouless energy $E_{\mathrm{Th}}=D / L^{2}$. The differential current noise $d S / d V$ is normalized to $4 e / 3 R_{N}$, i.e. twice the current noise in the normal state. It shows a similar reentrance effect as the conductance. The trivial effect of the energy dependent conductance on the noise is eliminated in the effective charge $q_{\text {eff }}=(3 / 2) d S / d I$. The dip in the effective charge below $E_{\mathrm{Th}}$ is therefore solely due to higher correlations. The right graph shows schematically the proximity wire.

multi-terminal structures with uncorrelated leads.

A natural question arising in this context is, what happens if the incoming particles are correlated. This can be achieved by using a superconducting terminal, from which particles are injected by Andreev reflection, i. e. as correlated electron-hole pairs. In a single-mode beam-splitter geometry this can eventually lead to positive crosscorrelationst. Some doubts have been raised, that the positive correlations survive in the many channel limit . Thus, we will study below one example of a superconducting beam splitter, in which large positive crgsscorrelations appear Similar results have been obtained in different geometries 19,11 .

\section{Diffusive Wire}

We study a two-terminal geometry, where a diffusive wire is mounted between a normal and a superconducting terminal, see the inset of Fig. 11. We expect a doubling of the effective charge, both in the quantum coherent regime at zero energy, and in the incoherent regime $E \gg E_{T h}$ (with Thouless energy $E_{T h}=D / L^{2}$ ). However, it is well know that the conductance is enhanced at intermediate energies due to the proximity effect - the so-called reentrant behaviour. To access the noise in this regime, we make use of an extended Keldysh Green's function method outlined in Ref. 12. Inside the mesoscopic wire the quasiclassical transport equations are obeyed 13 . In a diffusive normal metal wire they read

$$
D \frac{\partial}{\partial x}\left(\check{G}(x, \chi) \frac{\partial}{\partial x} \check{G}(x, \chi)\right)=\left[-i E \bar{\tau}_{3}, \check{G}(x, \chi)\right] .
$$

Here $D$ is the diffusion constant and $x$ the coordinate along the wire, which has a length $L$. It's conductance is $G_{\mathrm{N}}=\sigma A / L$ (cross section $A$ ). At both ends boundary conditions to reservoirs have to be supplied. At the normal end with ideal connection the Green's function is continuous: $\breve{G}(0, \chi)=\check{G}_{\mathrm{N}}(\chi)$. The currentcorrelations are encoded in the dependence on the so-called counting field $\chi$. The 
other end is connected to a superconducting reservoir by a contact of negligible resistance, which leads to the houndary condition $\check{G}(L)=\check{G}_{\mathrm{S}}$. Details of the calculation can be found in Ref.12.

In Fig. 11 we present results for the transport properties at zero temperature and for $E_{T h} \ll \Delta$. Both the differential current noise $d S / d V$ and the differential conductance $d I / d V$ show a reentrant behaviour. The energy dependence of both quantities is nonetheless different, demonstrating that the noise can not be simply obtained by multiplying a doubled energy-independent noise $4 e G_{N} V / 3$ by the differential resistance. Consequently this leads to an effective charge, defined by $q_{\mathrm{eff}}(V)=(3 / 2) d S / d I$, which drops below the value of $2 e$ at energies of the below $\approx 5 E_{T h}$. We believe this drop is due to a pair-pair correlation effect. This is supported by the fact the the suppression of the effective charge is mainly below the energy at which the maximal enhancement of the conductance occurs. A confirmation of this conjecture could be obtained in a system with two superconducting terminals, in which pair-pair correlations could be suppressed by destructive interference.

\section{Beam Splitter}

To address the question whether positive crosscorrelations can exist in a beam splitter geometry with one superconducting lead and two normal leads we study the layout shown in the inset of Fig. 2. The superconductor is held at zero voltage and the two normal terminals are biased symmetrically at the same voltage $V$. The terminals are connected by tunnel junctions to a central node. This could be either a small metallic island or a chaotic cavity and is assumed to be so large, that we can neglect charging effects. The transport properties are easily obtained using the circuit theory formulation 14,45 . In fact, for a setup where an arbitrary number of terminals is conpected to one common node by tunnel junctions the general solution can be obtained 9 . Note also, that this solution includes_not only the noise, but also the full counting statistics of this mesoscopic structure 16 .

We will now concentrate on auto- and crosscorrelations only. These are defined as $S_{i j}=2 \int d t\left\langle\delta I_{i}(t) \delta I_{j}(0)\right\rangle$, where $I_{i(j)}(t)$ are the time dependent currents in terminal $i(j)$. We obtain in the low-energy limit $(T=0, V \ll \Delta)$ :

$$
\begin{array}{r}
G=\frac{g_{S}^{2} g_{N}^{2}}{\left(g_{S}^{2}+g_{N}^{2}\right)^{3 / 2}} \quad, S=2 G|e V|\left(2-5 \frac{g_{S}^{2} g_{N}^{2}}{\left(g_{S}^{2}+g_{N}^{2}\right)^{2}}\right), \\
S_{12}=\frac{G|e V|}{2}\left(1-5 \frac{g_{S}^{2} g_{N}^{2}}{\left(g_{S}^{2}+g_{N}^{2}\right)^{2}}\right), S_{11}=\frac{G|e V|}{2}\left(3-5 \frac{g_{S}^{2} g_{N}^{2}}{\left(g_{S}^{2}+g_{N}^{2}\right)^{2}}\right) .
\end{array}
$$

For completeness we also cite the results for the case, in which the superconductor is in its normal state:

$$
\begin{gathered}
G^{N}=\frac{g_{S} g_{N}}{\left(g_{S}+g_{N}\right)} \quad, \quad S^{N}=2 G^{N}|e V|\left(1-2 \frac{g_{S} g_{N}}{\left(g_{S}+g_{N}\right)^{2}}\right), \\
S_{12}^{N}=-\frac{G^{N}|e V|}{2} \frac{g_{S} g_{N}}{\left(g_{S}+g_{N}\right)^{2}}, \quad S_{11}^{N}=\frac{G^{N}|e V|}{2}\left(2-3 \frac{g_{S} g_{N}}{\left(g_{S}+g_{N}\right)^{2}}\right) .
\end{gathered}
$$

T\%: submitted to World Scientific on November 21, 2018 


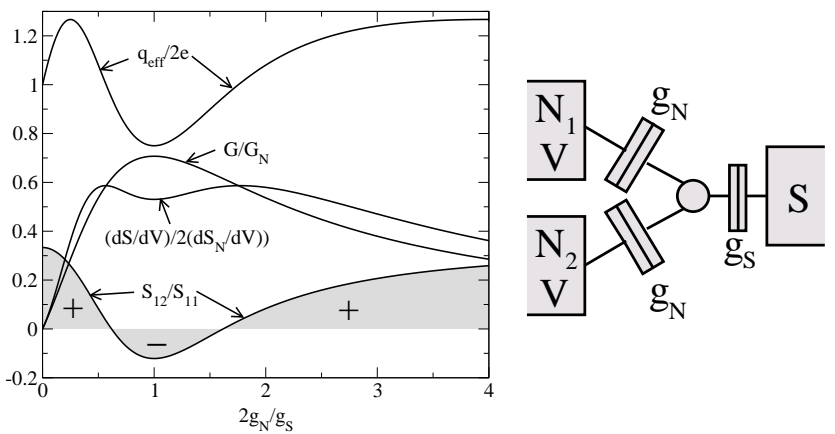

Figure 2. Transport properties of the beam splitter shown on the right. Remarkably, the crosscorrelation for the superconducting beam-splitter are positive in most of the parameter range. This is in contrast to the normal case, where the crosscorrelations are negative for the whole parameter range (not shown). Conductance and noise in the superconducting case (normalized to the normal case) show show a qualitative similar behaviour like the diffusive wire. However, the effective charge, here defined by the ratio $q_{\mathrm{eff}}=S I^{N} / S^{N} I$ shows a non-monotonic behaviour as function of the conductance ratio.

In Fig. 2 we summarize the transport properties, emphasizing the difference between the superconducting state and the normal state. Note that the total current noise is given by $S=2 S_{11}+2 S_{12}$. An interesting observation is that the transport properties are invariant under inversion of the conductance ratio $g_{N} / g_{S}$. In the following we will thus distinguish the case of weak (or strong) proximity effect determined by $g_{N} \not \approx g_{S}$ and the case of optimal proximity effect defined by $g_{N} \approx g_{S}$. Below we will use the terminology weak for both the weak and the strong proximity regimes equally.

The conductance vanishes rapidly in the limit of weak proximity and shows a resonance in the regime of optimal proximity. This actually defines the condition for optimal proximity effect: the regime, in which the proximity effect has the largest impact on the conductance. Note, that without proximity effect the conductance would vanish always in the present geometry. In Figure 2 we defined the effective charge in the superconducting case with respect to the normal case: $q_{\mathrm{eff}}=S I^{N} / S^{N} I$. This is in analogy to the diffusive wire, studied in the previous section. Remarkably, the effective charge shows an increase above $2 e$ for the case of weak proximity. Around the resonance condition $g_{S} \approx g_{N}$ the effective charge drops below $2 e$ and reaches a minimum of $(3 / 2) e$ for $g_{S}=g_{N}$. We note that this behaviour stems from our definition of the effective charge. The Fano factor for the superconducting case, defined by $F=S / 2 e I$, never increases above 2 .

The most surprising observation is that we can obtain positive crosscorrelations in the superconducting beam splitter, whereas they are manifestly negative for the normal beam splitter. Interestingly the positive crosscorrelations are found in the limit of weak proximity 11 , or in the absence of proximity 17 . Thus, they should be obtained from simple arguments. Indeed, from a calculation of the full counting statistics 8 , it follows that in the weak proximity limit the statistics consists of independent events of pair tunneling. The possible events are pair tunneling 
into terminal $N_{1}$ or $N_{2}$ and correlated tunneling in both terminals. These events occur with equal probabilities, however, in the limit of weak proximity these events are uncorrelated. Thus, two particle tunneling events into the same lead do not contribute to cross correlations. In contrast, tunneling of two particles into different leads is automatically positively correlated. Thus, it follows quite generally, that the crosscorrelations are positive.

Following this argumentation to the regime of optimal proximity effect in fact shows, that it is much harder to understand the occurrence of negative correlations in this regime. We propose the following interpretation. These negative crosscorrelations occur in the same regime, in which the effective charge drops below $2 e$. For uncorrelated electrons a suppression of the effective charge is interpreted as a consequence of the Fermi statistics. More precisely, it is a consequence of the Pauli principle, that two fermions can not occupy the same state. Now, the same holds obviously for pairs of electrons. If a two particle state is occupied by two electrons, this state is blocked for other pairs of electrons. Cooper pairs are such objects, and can not occupy the same state twice. Note, that we speak of Cooper pairs, but rather mean an correlated electron pair, which has entered the normal metal node. In the limit of weak proximity, the average occupation of a state on the central node with an electron pair is small, and the occupation does not matter, therefore. The same holds in the limit of strong proximity, where the state is nearly always occupied. This changes in the limit of optimal proximity, where the occupation approaches $1 / 2$. Just as in the case of Fermions, this leads to a strong suppression of the Fano factor.

To understand the negative crosscorrelations we follow the previous argumentation. The Pauli repulsion of Cooper pairs reduces the noise in the 'incoming' beam of Cooper pairs (similar to lowering the temperature in a fermionic beam). Let us, therefore, consider an incoming 'noiseless' beam of Cooper pairs, which is distributed equally among the possible outgoing states. The crosscorrelations can be written in terms of occupations of the outgoing states $n_{1,2}$ as $s_{12}=\left\langle n_{1} n_{2}\right\rangle-\left\langle n_{1}\right\rangle\left\langle n_{2}\right\rangle$. The possible outgoing states are $\left(n_{1}=2, n_{2}=0\right)$, $\left(n_{1}=0, n_{2}=2\right)$, and $\left(n_{1}=1, n_{2}=1\right)$, where the last state is doubly degenerate. The average occupation in terminal 1 is then $\left\langle n_{1}\right\rangle=(1 / 4) \times 2+(1 / 2) \times 1=1$, and the same for terminal 2. For the crosscorrelations we need $\left\langle n_{1} n_{2}\right\rangle$. The first two outgoing states do not contribute, whereas the third yields $(1 / 2) \times 1$. Collecting all terms we find $s_{12}=-1 / 2$, which is negative. The value of the crosscorrelations in Fig. 2 is only $-1 / 8$. This discrepancy is probably due to the finite backscattering in the double tunnel junction geometry. Nevertheless the qualitative behaviour is correct.

\section{Summary}

We have shown that current-correlations in multi-terminal heterostructures display a number of quite remarkable properties, which depend on the phase coherent nature of quantum transport. The proximity effect in a diffusive wire modifies the conductance and the noise in a nontrivial way. Both are enhanced at energies of the order of the Thouless energy, which divides coherent and incoherent Andreev 
transport. We have defined an effective charge by referencing to the noise in the normal state. The effective charge displays a suppression below $2 e$ for energies below $E_{T h}$, which could be due to coherent multiple Andreev-pair transport. For a multi-terminal structure with one superconducting and two normal metal arms, we have shown that positive crosscorrelations of the currents in the two arms are a generic feature in these system. It is remarkable, that in the region, in which the proximity effect has the strongest impact on the conductance, the crosscorrelations are negative. This can be explained by a Pauli exclusion principle for Cooper pairs in a transport process. Finally, we would like to emphasize the similarity of the noise behaviour in a diffusive wire and a multiple tunnel junction geometry. In particular, the suppression of the Fano factor in the regime of optimal proximity effect has probably the same origin. For the tunnel-junction geometry the finite occupation of the central node with Cooper pairs leads to a reduces Fano factor and positive cross correlations. In the diffusive wire the effective charge (equivalent to the Fano factor) are suppressed below energies of the order of $E_{T h}$, which is probably due to a finite occupation of the wire with Cooper pairs. A deeper understanding of this phenomenon is obviously interesting for further studies.

We acknowledge useful discussions with M. Büttiker, J. C. Cuevas, A. A. Kozhevnikov, D. E. Prober, B. Reulet, and P. Samuelsson. This work was supported by the Swiss NSF and the NCCR "Nanoscience".

\section{References}

1. Ya. M. Blanter and M. Büttiker, Phys. Rep. 336, 1 (2000).

2. V. A. Khlus, Sov. Phys. JETP 66, 1243 (1987); B. A. Muzykantskii and D. E. Khmelnitskii, Phys. Rev. B 50, 3982 (1994); M. J. M. de Jong and C. W. J. Beenakker, ibid. 49, 16070 (1994); K. E. Nagaev and M. Büttiker, ibid. 63, 081301(R) (2001).

3. A. A. Kozhevnikov et al., Phys. Rev. Lett 84, 3398 (2000).

4. X. Jehl et al., Nature 405, 50 (2000).

5. M. Henny et al., Science 284, 296 (1999); W. D. Oliver et al., Science 284, 299 (1999); S. Oberholzer et al., Physica (Amsterdam) E 6, 314 (2000).

6. M. Büttiker, Phys. Rev. Lett. 65, 2901 (1990).

7. T. Martin, Phys. Lett. A 220, 137 (1996); M. P. Anantram and S. Datta, Phys. Rev. B 53, 16390 (1996).

8. T. Gramespacher and M. Büttiker, Phys. Rev. B 61, 8125 (2000).

9. J. Börlin, W. Belzig, and C. Bruder, Phys. Rev. Lett. 88, 197001 (2002).

10. F. Taddei and R. Fazio, Phys. Rev. B 65, 134522 (2002).

11. P. Samuelsson, M. Büttiker, Phys. Rev. Lett. 88, 046601 (2002).

12. W. Belzig and Yu. V. Nazarov, Phys. Rev. Lett. 87, 067006 (2001).

13. A. I. Larkin and Yu. N. Ovchinnikov, Sov. Phys. JETP 26, 1200 (1968);

K. D. Usadel, Phys. Rev. Lett. 25, 507 (1970).

14. Yu. V. Nazarov, Superlattices Microst. 25, 1221 (1999).

15. Yu. V. Nazarov and D. Bagrets, Phys. Rev. Lett. 88, 196801 (2002).

16. Yu. V. Nazarov, Ann. Phys. (Leipzig) 8, SI-193 (1999).

17. P. Samuelsson and M. Buttiker, cond-mat/0207585 (unpublished). 